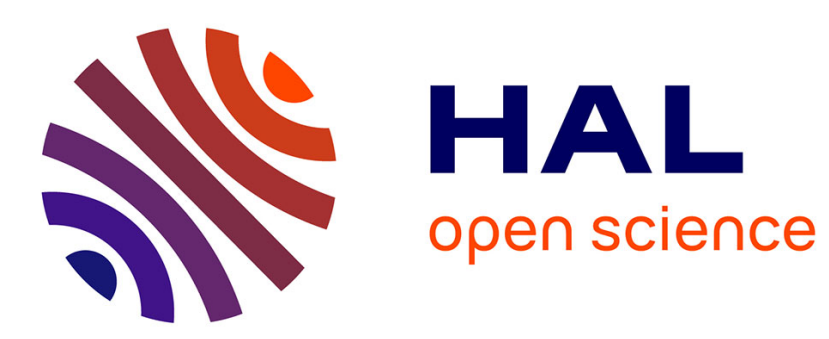

\title{
Marine Oil Slicks Quantification From L-band Dual-Polarization SAR Imagery
}

Olivier Boisot, Sébastien Angelliaume, Charles-Antoine Guérin

\section{To cite this version:}

Olivier Boisot, Sébastien Angelliaume, Charles-Antoine Guérin. Marine Oil Slicks Quantification From L-band Dual-Polarization SAR Imagery. IEEE Transactions on Geoscience and Remote Sensing, 2018, pp.1-11. 10.1109/TGRS.2018.2872080 . hal-01972394

\section{HAL Id: hal-01972394 \\ https://hal.science/hal-01972394}

Submitted on 7 Jan 2019

HAL is a multi-disciplinary open access archive for the deposit and dissemination of scientific research documents, whether they are published or not. The documents may come from teaching and research institutions in France or abroad, or from public or private research centers.
L'archive ouverte pluridisciplinaire HAL, est destinée au dépôt et à la diffusion de documents scientifiques de niveau recherche, publiés ou non, émanant des établissements d'enseignement et de recherche français ou étrangers, des laboratoires publics ou privés. 


\title{
Marine Oil Slicks Quantification From L-band Dual-Polarization SAR Imagery
}

\author{
Olivier Boisot, Sébastien Angelliaume, and Charles-Antoine Guérin
}

\begin{abstract}
We show, using simple physical models, that a quantitative estimation of the volume fraction of marine oil slicks can be achieved from dual-polarization Synthetic Aperture Radar (SAR) imagery. Volume fraction, which quantifies the proportion of seawater in oil in the case of a mixture, depends primarily on volume scattering mechanisms and is inferred from the Polarization Ratio in L-band. A quantification algorithm is derived, namely, the Volume Fraction Estimation (VFE) algorithm which is applied to two experimental datasets acquired in the Mediterranean Sea during the POLLUPROOF'2015 exercise, and in the North Sea during the NOFO'2015 experiment using the Office National d'Études et de Recherches Aérospatiales (ONERA) airborne L-band SETHI system. The resulting volume fraction maps of the quantification method are presented and discussed, opening new perspectives for marine oil slicks monitoring by means of dual-polarization SAR imagery.
\end{abstract}

Index Terms-ocean radar sensing, marine oil slick, quantification, volume fraction, complex effective permittivity, dualpolarization SAR, L-band

\section{INTRODUCTION}

A IRBORNE and spaceborne remote sensing sensors are commonly used by authorities and oil and gas companies to monitor hydrocarbons in the offshore domain [1]-[3]. The interest in remote sensing techniques lies not only in the global monitoring of the maritime environment to detect and track incidents or possible boat fuel releases, but also in the identification of the natural occurrence of crude oils (seeps) on the ocean surface, testifying to the presence of mature source rock on the seafloor [4]. Today state-of-the-art approaches for detecting oil slicks at sea are typically based on Synthetic Aperture Radar (SAR) imagery [5]-[7] which is not impacted by weather conditions as the optical imagery.

Marine oil slicks can be found under two primary forms, namely, surfactant films and crude oil slicks. Surfactant (surface-active agents) films are thin surface films made of amphiphilic organic compounds, consisting of a hydrophilic head group and a hydrophobic tail, which arrange themselves as mono-molecular films whose thickness is, typically, 2.4 $2.7 \mathrm{~nm}$ [8]. Their occurrence has been observed for sea surface wind speed $u_{10} \leq 5 \mathrm{~m} . \mathrm{s}^{-1}$ [9], otherwise, they are mixed and dissolved in the bulk water. When originating from natural sources, which is the most frequent case, they are referred to as biogenic or natural films. These films are mostly observed

O. Boisot and S. Angelliaume work within the Département d'Électromagnétisme et Radar (DEMR), ONERA, F-13661, Salon cedex Air, France.

C.-A. Guérin is with the Université de Toulon, Aix-Marseille Université, CNRS-INSU, IRD, Mediterranean Institute of Oceanography (MIO UM110), 83957 La Garde, France. in coastal and upwelling zones where marine fauna and flora activity is intense. Surfactant films can also be observed from accidental vegetable oil spills [10], [11] or at the edge of crude oil slicks due to weathering processes.

Crude oil slicks (including mineral oils, refined product of crude oils) are organic compounds constituted of alkanes, cycloalkanes and aromatics with preferentially hydrophobic character. When released at sea, crude oils are mixed with seawater under the action of wind and waves, resulting in a slick composed of a mixture of oil and water, such as emulsion, underneath an oil film. Oil and water emulsions are composed of small droplets of one medium in the background of the other, that is Water-in-Oil (W/O) or Oil-in-Water $(\mathrm{O} / \mathrm{W})$ emulsions. The most frequently encountered offshore emulsions are the $\mathrm{W} / \mathrm{O}$ case, but $\mathrm{O} / \mathrm{W}$ emulsions can also be found [12]. Droplets diameter of such emulsions is lower than $1 \mu \mathrm{m}$ in the case of fine emulsion, whereas larger than $1 \mu \mathrm{m}$ in the case of coarse emulsions [12]. In the case of W/O emulsion, the water content is generally between 50 and $75 \%$ [13]. Crude oil slicks form thick layers at the sea surface ranging typically from $\mu \mathrm{m}$ to $\mathrm{mm}$, but can reach $\mathrm{cm}$-range thickness in the case of fresh oil spill (accidents) and low sea state [8]. They originate mainly from anthropogenic sources, such as oil rigs, ships, and so on but also from natural seeps.

The impact of monolayer surfactants on the sea surface has been well investigated since the 1960s. Various aspects have been addressed such as physicochemical approaches [14] [16], experimental and laboratory studies [17]-[19] and the implications in remote sensing [20]-[24]. Even though the thickness of surfactant films is far smaller than that of crude oil slicks, their impact on the sea surface damping is of the same order of magnitude, which results in a damping of Bragg-wavelength capillary-gravity waves. Oil and seawater emulsions modify the dielectric properties of the remotely sensed surface, thus impacting the scattering coefficients of the scattering process. These two combined effects result in a global attenuation of the electromagnetic (EM) backscattered signal. As a result, marine oil slicks appear as dark patches in the SAR images, compared to the surrounding sea surface.

Essential requirements for global monitoring of oil at sea and efficient clean-up operations are the identification of the impacted area as well as its characterization (nature of oil) and its quantification (volume of oil). Many methods have been proposed in the last decades for the detection of oils on the ocean surface, many of them relying on SAR data (see e.g. [23] and [24] for a review). The most relevant radar parameters for marine oil slicks detection have been recently identified in [25] together with the most appropriate imaging mode in 
the context of marine pollution detection. Different studies have concentrated their efforts to characterize and discriminate oil spills from look-alike phenomena [26], [27] while others have investigated the mixing of oil and seawater from SAR sensors [28]-[30]. Up to now, only an oil/seawater mixing classification have been proposed, namely, the oil/seawater mixing index $M(-1 \leq M \leq 1)$ [28]. This index characterizes the oil and seawater mixing type by differentiating the origin of the attenuation of the backscattered signal in presence of an oil slick. Positive values indicate an attenuation mostly due to surface roughness damping from a surface film whereas negative values indicate an attenuation mostly due to a modification of the relative complex permittivity from a mixture of oil and seawater. This index has been used later in [30] where a methodology has been proposed based on dual co-polarized ( $H H$ and $V V$ ) SAR images to detect and quantify the relative concentration of pollutant on the ocean surface.

We present in this paper a novel methodology for the quantitative retrieval of the volume fraction $\left(f_{v}\right)$ of an oil and seawater mixture. The volume fraction estimation is mainly related to the effective complex permittivity of the underneath oil and seawater mixture and can be evaluated from the Polarization Ratio $(P R)$ in L-band together with the effective complex permittivity. The proposed methodology makes a combined utilization of models pertaining to the surface roughness, the complex permittivity and the scattering process at the sea surface. These models are reviewed in Section II. In Section III the quantification algorithm is introduced and its range of application discussed. A first application to experimental SAR data is presented in Section IV.

\section{PHySiCAL MODELING}

\section{A. Sea surface}

1) Spectral modeling of sea surface: One of the most popular sea surface wavenumber spectrum models today is the unified directional spectrum proposed by Elfouhaily et al. [31], which has been designed to address the complete range of wave scales and sea states. It combines various theoretical and experimental results in order to derive a directional sea wavenumber spectrum taking into account a wide range of sea wavelength, ranging from gravity to capillarity waves. The spreading function describing its azimuthal dependency is limited to the first even Fourier harmonic:

$$
\Psi\left(k, \phi_{k}\right)=\frac{\Psi_{0}(k)}{2 \pi k}\left[1+\Delta(k) \cos \left(2\left(\phi_{k}-\phi_{w}\right)\right)\right]
$$

where $\left(k, \phi_{k}\right)$ are the polar coordinates of the sea surface wavenumber, the function $\Delta(k)$ is defined as the ratio of the upwind/crosswind directional spectrum and $\phi_{w}$ is the wind direction.

2) Seawater complex permittivity: One important descriptive parameter for seawater in presence of oil is the complex relative dielectric permittivity:

$$
\varepsilon=\varepsilon^{\prime}+\mathrm{i} \varepsilon^{\prime \prime}
$$

It is customary to refer to the real part $\varepsilon^{\prime}$ as the relative permittivity and to the imaginary part $\varepsilon^{\prime \prime}$ as the loss factor. Seawater is a dispersive medium with high values of relative permittivity and loss factor. A recent model, based on the classical DoubleDebye Dielectric Model and adjusted to microwave satellite data can be found in [32], and examples are provided in table I.

3) Thermophysical properties of seawater: Thermophysical properties of seawater are quite similar to those of pure water. However, dissolved salt in water makes a difference which must be taken into account in modeling the interaction of seawater and oils mixture. A review of thermophysical properties has been provided by Sharqawy et al. [34], where many models have been updated. Examples can be found in table I.

\section{B. Impact of oil at sea}

1) Sea roughness damping model: It was the Italian physicist Marangoni [35] who first realized that wave damping from viscous surface films on seawater is due to the change in surface tension caused by waves motion. Later on, Cini and Lombardini [15] were the first to formalize the effect of resonance-type damping in the short-gravity waves region, also called as the Marangoni effect, from mono-molecular surfactant films. A review of the damping effect can be found in [36]. Denoting $\Delta_{\text {oil }}$, the viscous coefficient of the sea surface covered by a mono-molecular surfactant film and $\Delta_{s w}$, the filmfree sea surface, the viscous damping ratio is [36]:

$$
y(k)=\frac{\Delta_{\text {oil }}}{\Delta_{s w}}=\frac{1+X(\cos \beta-\sin \beta)+X Y-Y \sin \beta}{1+2 X(\cos \beta-\sin \beta)+2 X^{2}}
$$

where:

$$
X=\frac{|E| k^{2}}{\sqrt{2 \omega_{s w}^{3} \eta_{s w} \rho_{s w}}} \quad \text { and } \quad Y=\frac{|E| k}{4 \omega_{s w} \eta_{s w}}
$$

with $E=-|E| \exp (\mathrm{i} \beta)$ the complex dilatational elasticity modulus of the oil film (with $|E|$ in $\left[\mathrm{N} . \mathrm{m}^{-1}\right]$ and $\beta$ the phase angle), $k$ the wavenumber of waves, $\omega_{s w}=\sqrt{g k+\sigma_{s w} / \rho_{s w} k^{3}}$ the capillarity-gravity waves dispersion relationship, $g$ the gravity constant at sea level, $\eta_{s w}, \rho_{s w}$ and $\sigma_{s w}$, the dynamic viscosity, the volumetric mass and the surface tension of seawater, respectively. Table II recaps some values of the complex dilatational modulus $E$, taken from [33], for some crude oils. Other examples, in the case of biogenic slicks, can be found in [36].

Further calculations of the damping ratio induced by a viscous surface film in the case of finite thickness film have been conducted by Jenkins and Jacobs [37]. It has been shown that expression (3) is the limiting case of a zero thickness film, holding for thickness film smaller than $0.1 \mathrm{~mm}$ in general. Its also holds for surface layers smaller than $1 \mathrm{~mm}$ under the assumption that the kinematic viscosity ratio $\left(v_{\text {oil }} / v_{s w}\right)$ remains smaller than 100 [37]. However, this last assumption is not verified for the majority of known oils, due to a very large variation of viscosity values from one oil to another [13]. Nevertheless, an oil layer at sea is quickly spread and mixed by weathering processes such as natural wind stress and waves motion. One can thus assume that the condition of an oil layer smaller than $0.1 \mathrm{~mm}$ is satisfied in a majority of cases, except perhaps in the specific case of fresh release of crude oils by low sea state. Expression (3) of the viscous damping ratio can 


\begin{tabular}{|c|c|c|c|c|c|c|c|}
\hline \multirow{2}{*}{$\begin{array}{c}\text { Frequency Band } \\
{\left[f_{E M}\right](\mathrm{GHz})}\end{array}$} & \multicolumn{4}{|c|}{ seawater properties $\left(S S T=10^{\circ} \mathrm{C} ; S A L=35 \mathrm{PSU}\right)$} & \multicolumn{2}{|c|}{ crude oil slick (from [33]) } & \multirow{2}{*}{$\begin{array}{c}\text { W/O mixture } \\
\varepsilon_{e f f} @ f_{v}=0.5\end{array}$} \\
\hline & $\varepsilon_{s w}$ & $\eta_{s w}[\mathrm{mPa} . \mathrm{s}]$ & $\rho_{s w}\left[\mathrm{~kg} \cdot \mathrm{m}^{-3}\right]$ & $\sigma_{s w}\left[\mathrm{mN} \cdot \mathrm{m}^{-1}\right]$ & $|E|\left[\mathrm{mN}^{\prime} \mathrm{m}^{-1}\right]$ & $\beta\left[^{\circ}\right]$ & \\
\hline $\mathrm{L}[1]$ & $74.77+73.71 \mathrm{i}$ & & & & & & $23.19+18.83 \mathrm{i}$ \\
\hline $\begin{array}{l}\text { C [5] } \\
X[10]\end{array}$ & $\begin{array}{l}66.45+36.78 \mathrm{i} \\
49.81+40.44 \mathrm{i}\end{array}$ & 1.397 & 1027 & 74.89 & 22.9 & -165.5 & $\begin{array}{l}20.88+9.507 \mathrm{i} \\
16.65+10.65 \mathrm{i}\end{array}$ \\
\hline
\end{tabular}

Table I: Examples of seawater thermophysical properties as well as oil slick parameters and W/O mixture effective complex permittivity as function of the frequency band.

\begin{tabular}{c|c|c|c}
\hline Oil number & Oil origin & $|E|\left[\mathrm{mN} \cdot \mathrm{m}^{-1}\right]$ & $\beta\left[{ }^{\circ}\right]$ \\
\hline \hline 1 & West Africa & 12.2 & -166 \\
\hline 2 & West Africa & 9.3 & -161.1 \\
\hline 3 & North Sea & 22.9 & -165.5 \\
\hline 4 & North Sea & 32.9 & -166.5 \\
\hline 5 & France & 14.4 & -165.6 \\
\hline
\end{tabular}

Table II: Some values of complex dilatational elasticity moduli of crude oils [33].

therefore be used for general marine oil slicks, and not only for monolayer surfactants such as biogenic slicks.

The viscous damping ratio $y$ can be related to the sea spectrum damping ratio $D_{\Psi}$ if one assumes that surface films are partially dispersed by wind stress and waves motion, resulting in a partial film coverage of the surface. Introducing a surface fraction factor $f_{s}$ to quantify the area covered by a film among the total sea surface area impacted by the slick $\left(0 \leq f_{s} \leq 1\right)$, the sea spectrum damping ratio is given by [38]:

$$
D_{\Psi}(k)=\frac{\Psi_{s w}(k)}{\Psi_{o i l}(k)}=\frac{1}{1-f_{s}+f_{s} / y(k)},
$$

with $\Psi_{s w}$ standing for the film-free sea wavenumber spectrum and $\Psi_{\text {oil }}$ the film-covered sea wavenumber spectrum.

2) Oils complex permittivity: Oils are dielectric media with low relative permittivity and very small loss factor. In the microwave range, going from $\mathrm{L}$ to $\mathrm{X}$-band, oils are a quasi non-dispersive medium; their relative pemittivity ranges from about 2.2 to 2.3 and their loss factor is about 0.01 [39], [40]. We will adopt the following value of the oil complex permittivity, which is valid in the frequency range $1-10 \mathrm{GHz}$ :

$$
\varepsilon_{o i l} \simeq 2.25+0.01 \mathrm{i}
$$

3) Effective complex permittivity of a mixture: Both W/O and $\mathrm{O} / \mathrm{W}$ emulsions can be found at sea [12], so that inclusions and background environment can be inverted, with no a priori knowledge of the mixture type. In the case of homogeneous spherical inclusions $\left(\varepsilon_{i}\right)$ in a homogeneous environment $\left(\varepsilon_{e}\right)$ with a volume fraction $f_{v}=V_{i} / V_{e}$, the classical Bruggeman formula [41] is the most adapted as it does not assume one medium or the other to be the background environment. It provides an explicit formula for the effective complex permittivity of the mixture:

$$
\left\{\begin{aligned}
\varepsilon_{e f f}= & \frac{1}{4}\left\{\varepsilon_{e}-\left(1-3 f_{v}\right)\left(\varepsilon_{i}-\varepsilon_{e}\right)+\cdots\right. \\
& \left.\sqrt{\left[\varepsilon_{e}-\left(1-3 f_{v}\right)\left(\varepsilon_{i}-\varepsilon_{e}\right)\right]^{2}+8 \varepsilon_{i} \varepsilon_{e}}\right\} \\
f_{v}= & \frac{\left(\varepsilon_{e f f}-\varepsilon_{e}\right)\left(\varepsilon_{i}+2 \varepsilon_{e f f}\right)}{3 \varepsilon_{e f f}\left(\varepsilon_{i}-\varepsilon_{e}\right)}
\end{aligned}\right.
$$

Examples of W/O mixture effective complex permittivity as function of the EM band are given in table I.

\section{Surface scattering}

1) Splitting rule: In order to distinguish volume and surface scattering effects, Guérin and Sentenac [42] derived a so-called splitting rule:

"The incoherent intensity of a composite medium with a rough interface is the sum of the incoherent intensity of a rough homogeneous surface with an effective permittivity and the incoherent intensity of the same composite medium below a flat interface. The coherent intensity is merely that of the rough effective homogeneous surface".

Thus, volume effects can be associated to the effective complex permittivity of the mixture in the water column and surface effects to the roughness of the surface, considering the effective complex permittivity of the homogeneous medium at the interface.

2) The Universal Weighted Curvature Approximation: There exists a wealth of surface scattering models to describe the interaction of EM wave with the sea surface [43]. The key parameters for radar observation, which depend on the viewing angles (namely, the incidence angle $\theta$ and the azimuth angle $\phi)$ as well as the surface roughness, are the Normalized Radar Cross Section (NRCS, $\sigma_{p p}^{0}$ ) and the $P R$, among others:

$$
P R=\frac{\sigma_{H H}^{0}}{\sigma_{V V}^{0}}, \quad 0<P R \leq 1
$$

where $\sigma_{H H}^{0}$ and $\sigma_{V V}^{0}$ are the co-polarized horizontal and vertical NRCS, respectively. As usual, the choice of a model results from a trade-off between simplicity and performance. Asymptotic surface scattering models, such as the classical Small Perturbation Method (SPM) [44], [45], the Kirchhoff Approximation (KA) [46] or the first-order Small Slope Approximation (SSA) [47]-[49] can be used in some specific conditions (low incidence angles, small roughness, etc) but predict a purely geometrical angular variation of the $P R$ and thus cannot account for their roughness dependence. Nontrivial $P R$ s and a better reproduction of the azimuthal behavior of the NRCS can be obtained with the family of Two-Scale Models, which account for the tilting effects of large scale waves onto small ripples [45], [50], [51]. Improved scattering models can also be obtained with functional or perturbative expansions with respect to an elevation, a slope or a curvature parameter such as the second-order SSA (SSA2) [48] or models taking explicitly into account the local surface curvature [52]-[58]. Among these models, which are more complex to use than the classical asymptotic models, we adopted one simple unifying model which has been found to address the 
variety of scales at the sea surface and to describe properly the roughness dependence of the $P R$ while being well adapted to the inversion of oceanic parameters, namely the so-called Universal Weighted Curvature Approximation (UWCA), first introduced in [57]. This model depends only on the sensor viewing angles and the sea surface wave number spectrum. Its expression for the co-polarized NRCS is given by:

$$
\sigma_{p p}^{0}=4 \pi\left|\mathbb{B}_{p p}\right|^{2} \Psi\left(\mathbf{Q}_{\mathbf{H}}\right)+|\mathbb{K}|^{2}\left[I_{s}-4 \pi \Psi\left(\mathbf{Q}_{\mathbf{H}}\right)\right]
$$

where:

$$
I_{s}=\frac{1}{\pi Q_{z}^{2}} \int_{\mathbb{R}^{2}} \mathrm{e}^{-\mathrm{i} \mathbf{Q}_{\mathbf{H}} \cdot \mathbf{r}}\left[\mathrm{e}^{-Q_{z}^{2}[\rho(\mathbf{0})-\rho(\mathbf{r})]}-\mathrm{e}^{-Q_{z}^{2} \rho(\mathbf{0})}\right] d \mathbf{r},
$$

is proportional to the classical Kirchhoff integral, $\mathbf{Q}_{\mathbf{H}}$ and $Q_{z}$ are the horizontal and vertical projections of the Ewald vector: $\mathbf{Q}=\mathbf{Q}_{\mathbf{H}}+Q_{z} \hat{\mathbf{z}}, \rho$ is the spatial autocorrelation function of the sea surface and $\Psi\left(\mathbf{Q}_{\mathbf{H}}\right)$ is the sea wavenumber spectrum taken at the Bragg wavenumber:

$$
\left\|\mathbf{Q}_{\mathbf{H}}\right\|=2 K_{0} \sin \theta=: K_{B},
$$

with $K_{0}$ being the EM wavenumber. The expression of the Bragg $\left(\mathbb{B}_{p p}\right)$ and Kirchhoff $(\mathbb{K})$ kernels can be found e.g. in [43]. In the following, we introduce the relative roughness coefficient $\Gamma$ :

$$
\Gamma(\theta, \phi)=\frac{4 \pi \Psi\left(\mathbf{Q}_{\mathbf{H}}\right)}{I_{s}},
$$

which quantifies the proximity to a Bragg scattering mechanism. This coefficient varies from 0 (pure facet reflections) to 1 (pure Bragg resonance mechanisms). We may therefore rewrite eq. (9):

$$
\sigma_{p p}^{0}=I_{s}\left[\Gamma\left|\mathbb{B}_{p p}\right|^{2}+(1-\Gamma)|\mathbb{K}|^{2}\right],
$$

leading to the following expression for the $P R(8)$ :

$$
P R=\frac{\Gamma\left|\mathbb{B}_{H H}\right|^{2}+(1-\Gamma)|\mathbb{K}|^{2}}{\Gamma\left|\mathbb{B}_{V V}\right|^{2}+(1-\Gamma)|\mathbb{K}|^{2}}=P R(\theta, \phi, \varepsilon)
$$

In this form, the azimuthal variations of the $P R$ are clearly controlled by the relative roughness $\Gamma$ defined in eq. (12).

\section{QUANTIFICATION METHOD}

The developed quantification method aims at estimating the volume fraction $f_{v}$ of an oil and seawater mixture, which quantifies the proportion of seawater in oil. The respective contributions of volume and surface modifications can be separated, owing to the aforementioned splitting rule. The estimation algorithm, referred to as Volume Fraction Estimation (VFE) algorithm, relies on the $P R$ properties in L-band and the comparison of the slick-impacted area with the surrounding slick-free seawater, used as an absolute reference. In the case of SAR images with complete slick coverage, the method could still be applied using a sea surface wave spectrum model to estimate the slick-free seawater NRCS but would provide qualitative results only, due to the unknown actual roughness of the sea surface.

As seen in eq. (14), the $P R$ at a given angle of incidence depends essentially on the relative roughness coefficient of the surface $(\Gamma)$ and the complex relative permittivity of the medium $(\varepsilon)$. The different $P R$ s, depending on the configuration of the composite medium can be summarized as:

$$
\begin{aligned}
& P R_{s w}=P R\left(\Gamma_{s w}, \varepsilon_{s w}\right) \\
& P R_{\text {film }}=P R\left(\Gamma_{f i l m}, \varepsilon_{s w}\right) \\
& P R_{\text {mix }}=P R\left(\Gamma_{s w}, \varepsilon_{\text {eff }}\right) \\
& P R_{\text {slick }}=P R\left(\Gamma_{\text {film }}, \varepsilon_{\text {eff }}\right)
\end{aligned}
$$

where the "sw", "film", "mix" and "slick" subscripts stand for, respectively, the pure seawater, the pure viscous surface film, the pure $\mathrm{W} / \mathrm{O}$ mixture and oil slick (surface film + mixture) case. $\varepsilon_{e f f}$ is the effective complex permittivity of a W/O mixture. We expect the $P R$ of film-covered areas $\left(P R_{\text {film }}\right)$ to depend explicitly on the underneath seawater complex permittivity due to the very small thickness of those surface films (from $\mathrm{nm}$ to $\mathrm{mm}$, which is small compared to the penetration depth of the microwaves in pure oil [25]). Note also, the assumption regarding the $P R$ of a W/O mixture $\left(P R_{\text {mix }}\right)$ is not completely true as an emulsion can have some damping effects on the sea roughness. However, as measured in [33], a fresh release of a pure W/O emulsion in seawater has a quasinegligible viscous damping effect. Stronger viscous damping effects originate from the formation of a pure viscous film above the sea surface during weathering processes, resulting in oil slick.

Sea roughness damping by a surface film tends to decrease the $P R$ while on the contrary a W/O mixture tends to increase it [25]. However, in L-band, the former effect is negligible since the $P R$ is dominated by the Bragg mechanism at a resonant wavelength which is little affected by the smallscale damping process. This is illustrated in Figure 1 where calculations of the $P R$ have been carried out in the upwind direction with the UWCA scattering model (eq. (14)) and an Elfouhaily spectrum for a wind speed $u_{10}=7 \mathrm{~m} . \mathrm{s}^{-1}$. In both $\mathrm{X}$ and L-band, the W/O mixture has a significant impact on the $P R$ while a surface viscous film only affects the X-band. Hence, the L-band relative roughness parameter of the surface film can be assumed unchanged with respect to its seawater counterpart,

$$
\Gamma_{f i l m} \simeq \Gamma_{s w}
$$

and the same holds for the corresponding PRs:

$$
P R_{\text {slick }} \simeq P R\left(\Gamma_{\text {sw }}, \varepsilon_{\text {eff }}\right)=P R_{\text {mix }}
$$

This shows that volume scattering taken into account by the effective complex permittivity has the dominant impact on the $P R$ in L-band while roughness damping effects are negligible. In the case of a seawater area impacted by a pure viscous surface film, the observed $P R$ in L-band $\left(P R_{f i l m}\right)$ will thus appear similar to that of the surrounding seawater area $\left(P R_{s w}\right)$. An illustration of this phenomenon can be seen on [Figure 13 (d), [25]] for real L-band SAR images recorded during the POLLUPROOF'2015 exercise developed later in this paper (see section IV-A). With the use of L-band copolarized SAR data, the VFE algorithm runs as follows:

1) Estimation of the seawater complex permittivity $\left(\varepsilon_{s w}\right)$ from external data of sea surface temperature $(S S T)$ and salinity $(S A L)$, 


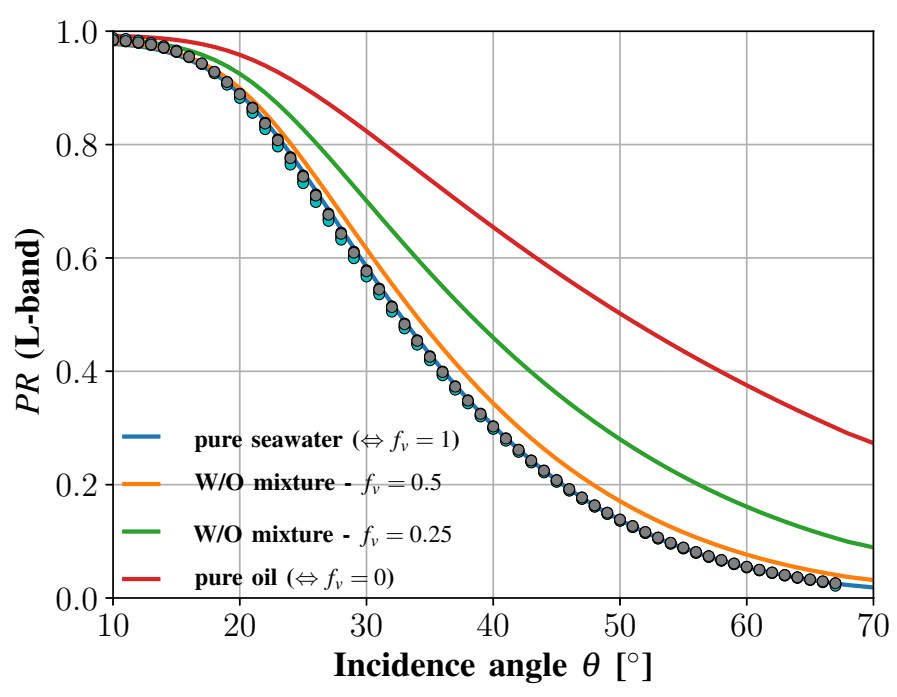

(a) L-band polarization ratio

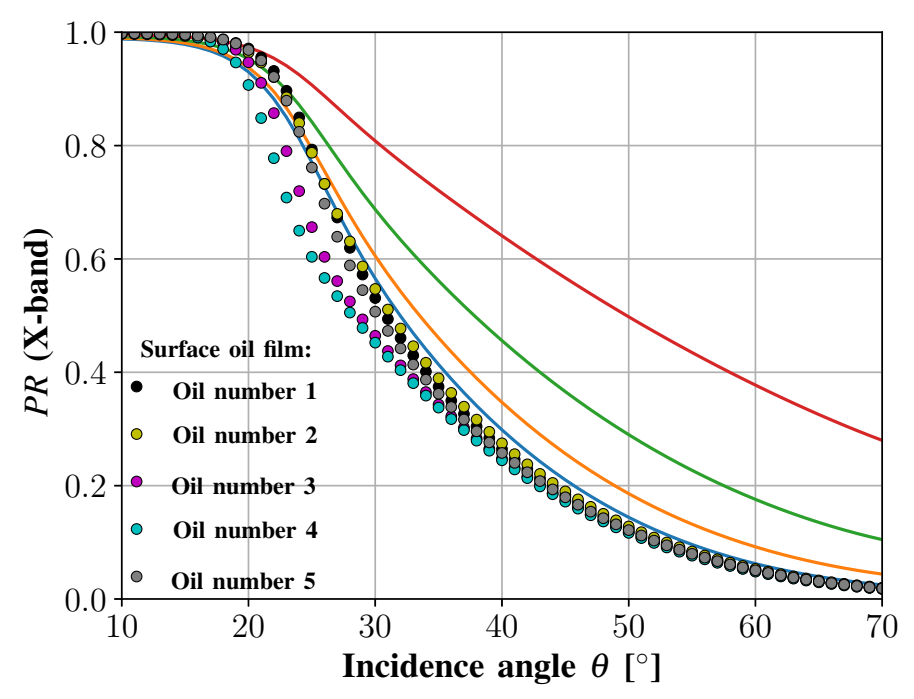

(b) X-band polarization ratio

Figure 1: Polarization ratio as a function of the incidence angle in (a) L-band (1 GHz) and (b) X-band (10 GHz) for different types of surface: pure sea water (solid blue line), W/O mixtures (solid orange and green lines) and pure oil (red line). The calculation has been performed in the upwind direction with the UWCA scattering model using an Elfouhaily spectrum with wind speed $u_{10}=7 \mathrm{~m} \cdot \mathrm{s}^{-1}$. Colored dots depict the case of a dampened sea roughness induced by a viscous surface oil films above the sea surface for a surface fraction of 1 and different values of complex dilatational modulus $E$, whose corresponding oil numbres are taken from Table II. In (a) L-band, all colored dots are superimposed. The seawater properties have been calculated for $S S T=10^{\circ} \mathrm{C}$ and $S A L=35 \mathrm{PSU}$ and can be found in Table I.

2) Estimation of the mean seawater relative roughness parameter $\left(\Gamma_{s w}\right)$ from the mean seawater polarization ratio $\left(P R_{s w}\right)$ and the seawater complex permittivity $\left(\varepsilon_{s w}\right)$ on the largest available area, as a function of the sensor incidence angle:

$$
\Gamma_{s w}=\left[1+\frac{P R_{s w}\left|\mathbb{B}_{H H}\left(\varepsilon_{s w}\right)\right|^{2}-\left|\mathbb{B}_{V V}\left(\varepsilon_{s w}\right)\right|^{2}}{\left|\mathbb{K}\left(\varepsilon_{s w}\right)\right|^{2}\left(1-P R_{s w}\right)}\right]^{-1}
$$

3) Joint estimation of the effective complex permittivity $\left(\varepsilon_{e f f}\right)$ and volume fraction $\left(f_{v}\right)$ of the W/O mixture by solving the system:

$$
\left\{\begin{aligned}
P R\left[\Gamma_{s w}, \varepsilon_{e f f}\right] & =P R_{\text {data }} \\
\operatorname{Re}\left[\varepsilon_{e f f}\right] & =\operatorname{Re}\left[\varepsilon_{e f}^{\text {Brug }}\left(\varepsilon_{s w}, \varepsilon_{\text {oil }}, f_{v}\right)\right] \\
\operatorname{Im}\left[\varepsilon_{e f f}\right] & =\operatorname{Im}\left[\varepsilon_{\text {eff }}^{\text {Brug }}\left(\varepsilon_{s w}, \varepsilon_{\text {oil }}, f_{v}\right)\right]
\end{aligned}\right.
$$

where the effective complex permittivity used in the first line of (19) is constrained by the mixing rule of Bruggeman (eq. (7)). This leads to a nonlinear system of 3 equations with 3 unknown parameters, which can be solved numerically with classical solvers. The solution is unique in view of the monotonic behavior of $P R\left[\varepsilon_{e f f}\right]$, $\operatorname{Re}\left[\varepsilon_{e f f}^{\text {Brug }}\left(f_{v}\right)\right]$, and $\operatorname{Im}\left[\varepsilon_{e f f}^{\text {Brug }}\left(f_{v}\right)\right]$.

\section{APPLICATION TO EXPERIMENTAL DATA}

\section{A. Data sets presentation}

The methodology presented in this paper has been applied to experimental SAR data collected with SETHI (the airborne remote sensing system developed by ONERA [59]) during

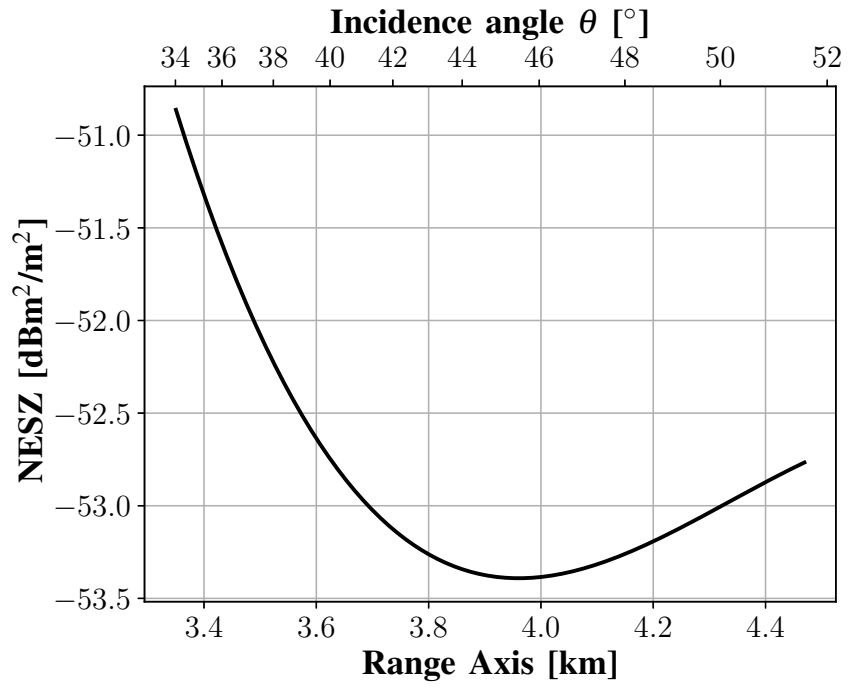

Figure 2: SETHI L-band instrument noise floor (NESZ) as function of the radar range and sensor incidence angle for POLLUPROOF'2015 and NOFO'2015 experiments

two offshore oil-on-water exercises: POLLUPROOF'2015 and NOFO'2015.

The POLLUPROOF'2015 experiment (18 and 22 May 2015) was conducted over the Mediterranean Sea (off the French coast, near $42^{\circ} 45.5^{\prime} \mathrm{N}, 5^{\circ} 48.5^{\prime} \mathrm{E}$ ) and focused on the release and subsequent observation of several Hazardous and Noxious Substances (HNS). The main goal of this experiment was to establish a procedure for collecting evidence of illegal 


\begin{tabular}{|c|c|c|c|c|c|c|c|c|c|}
\hline Experiment & $\begin{array}{c}\text { Time of } \\
\text { release (UTC) }\end{array}$ & $\begin{array}{l}\text { Amount of } \\
\text { release }\left[\mathrm{m}^{3}\right]\end{array}$ & $\begin{array}{l}\text { Released } \\
\text { substance }\end{array}$ & $\begin{array}{c}\text { Time of } \\
\text { imaging (UTC) }\end{array}$ & $\begin{array}{c}\text { Wind speed }(10 \mathrm{~m}) \\
u_{10}\left[\mathrm{~m} . \mathrm{s}^{-1}\right]\end{array}$ & $\begin{array}{l}\text { Wind direction } \\
\text { (from) } \phi_{w}\left[{ }^{\circ}\right]\end{array}$ & $\begin{array}{c}\text { Radar look } \\
\text { direction } \phi\left[^{\circ}\right]\end{array}$ & $\begin{array}{l}S S T^{a} \\
{\left[{ }^{\circ} \mathrm{C}\right]}\end{array}$ & $\begin{array}{l}S A L^{a} \\
{[\mathrm{PSU}]}\end{array}$ \\
\hline $\begin{array}{l}\text { POLLU- } \\
\text { PROOF }\end{array}$ & $\begin{array}{l}15: 01-15: 28 \\
15: 24-15: 40\end{array}$ & 1 & $\begin{array}{l}\text { Rapeseed oil } \\
\text { FAME }\end{array}$ & $16: 07$ & 8.2 & 270 & 181 & 15.1 & 38.08 \\
\hline NOFO & $06: 30-08: 00$ & 45 & Mineral oil & 10:01 & 5.1 & 270 & 22 & 9.49 & 35.16 \\
\hline
\end{tabular}

${ }^{a}$ values taken from Copernicus Marine website: http://marine.copernicus.eu.

Table III: Environmental conditions and properties of released substance.

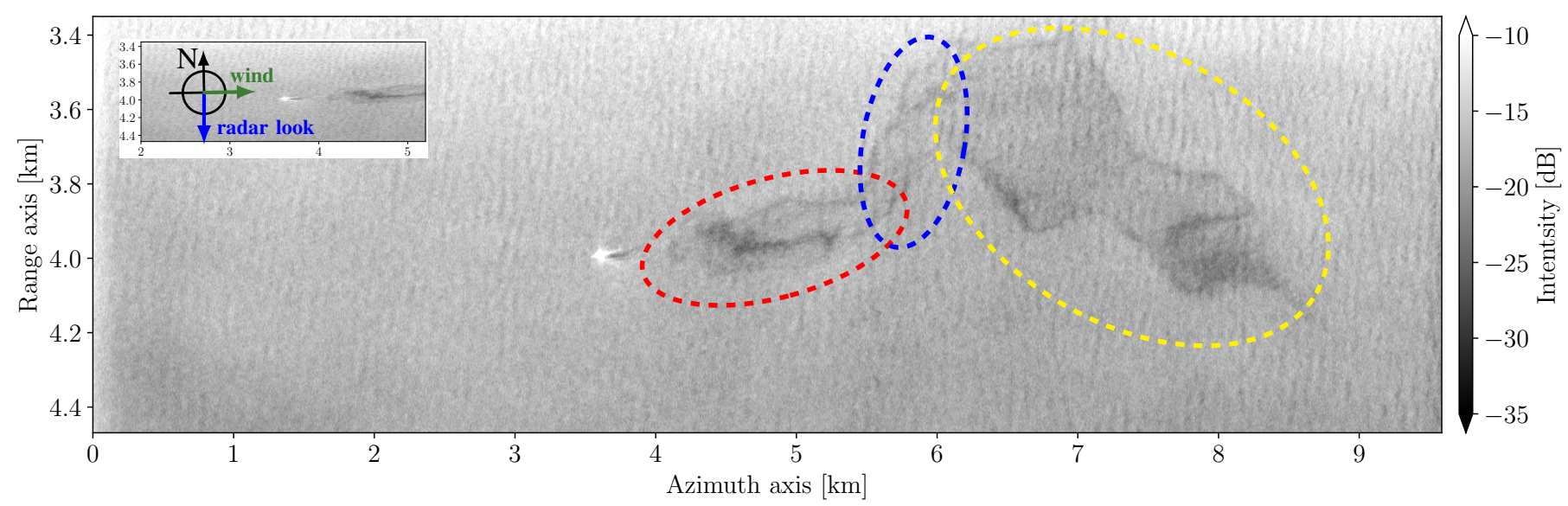

(a) POLLUPROOF'2015

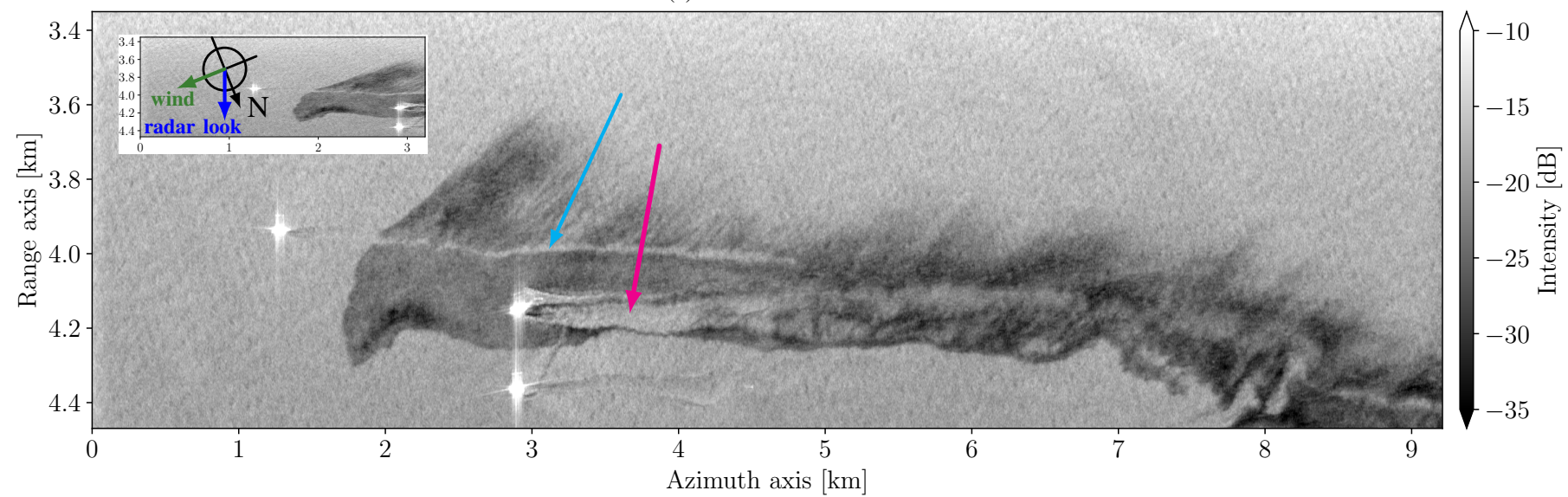

(b) NOFO'2015

Figure 3: L-band $V V$-polarization intensity images recorded during the (a) POLLUPROOF'2015 and (b) NOFO'2015 experiments. A 7x7 multilook processing has been applied. Thumbnails of corresponding images in an orthonormal basis are superimposed showing the geographical North (black arrow), the radar look direction (blue arrow) and the wind blowing direction (green arrow). (a) The yellow and red ellipses correspond to the successive spills of rapeseed oil and FAME, respectively. In between, a mixture of both products is formed and delimited by a blue ellipse. (b) The magenta and cyan arrows show the relatively clean sea left behind the MOS Sweeper mechanical recovery boom and the wake left behind the crossing of a ship through the slick, respectively.

marine pollution by HNS from airborne sensors [30]. In the following, we will focus on the 22 May exercise where $1 \mathrm{~m}^{3}$ of rapeseed oil (colza oil) and $1 \mathrm{~m}^{3}$ of Fatty Acid Methyl Esters (FAME, biofuel directly added in conventional fuels) were released at sea within a small time lag (see Table III). Rapeseed oil is classically used to simulate biogenic films on the sea surface and has already been imaged by SAR sensors [26], [60], [61] whereas FAME forms a cloud of microdroplets in the water column [30].

The NOFO'2015 experiment (8-14 June 2015) is an oil spill cleanup exercise managed by the Norwegian Clean Seas Asso- ciation for Operating Companies (NOFO). It was carried out in the North Sea (230 km NorthWest of Stavanger, Norway) within 10 Nautical Miles of position ( $\left.59^{\circ} 59^{\prime} \mathrm{N}, 02^{\circ} 27^{\prime} \mathrm{E}\right)$. In the following, we will focus on the 09 June exercise during which the MOS Sweeper mechanical recovery boom [62] was tested at sea [25]. For this experiment, the released product was an emulsion of water in mineral oil with a water content of $60 \%$. It consisted of a mixture of water, Oseberg crude oil and a small addition of IFO 380 (Intermediate Fuel Oil or marine diesel oil, with viscosity of $380 \mathrm{~mm}^{2} . \mathrm{s}^{-1}$ ). For the trial, $45 \mathrm{~m}^{3}$ of emulsion were discharged at sea. Assuming the 
entire volume of hydrocarbon was spread on the surface, the upper limit of the average thickness of the slick is about 1 $\mu \mathrm{m}[25]$.

Meteorological informations were obtained from Météo-France, the French national meteorological center (POLLUPROOF'2015) and the Norwegian Meteorological Institute (NOFO'2015) while sea surface thermophysical characteristics were obtained from Copernicus Marine website (see Table III).

During both exercises, quad-polarimetric SAR (POLSAR) data were collected with SETHI at L-band with a range resolution of $1 \mathrm{~m}$ (bandwidth from 1.25 to $1.4 \mathrm{GHz}$ ). Images were processed with an azimuth (along-track) resolution equal to the range resolution. The imaged area was about $9.2 \mathrm{~km}$ in azimuth and $1.1 \mathrm{~km}$ in range, with incidence angles spanning from $34^{\circ}$ to $52^{\circ}$. The L-band SAR sensor which operated onboard SETHI is characterized by a very low instrument noise floor, allowing a sufficiently high Signal-to-Noise Ratio (SNR) over both slick-free and slick-covered areas for valid analysis of surface characteristics.

The SNR is a crucial parameter in the context of marine oil slicks sensing with SAR and its impact must be carefuly taken into account. As a matter of fact, the co-polarized coherency has been claimed to decrease rapidly over slickcovered surface [24] but it was later suggested in [61] and demonstrated in [25] that this is mainly due to instrumental noise decorrelation. However, providing a sufficiently high SNR, it has been shown that the co-polarized coherency parameter is not impacted by oil slicks [25], [29] and is therefore a useful parameter to eliminate ships and/or SAR processing artifacts, as was done with the present data sets.

For the SETHI instrument, the Noise Equivalent Sigma Zero (NESZ) estimated using the method proposed in [63] is very low, ranging from about -53.5 to $-51 \mathrm{dBm}^{2} / \mathrm{m}^{2}$ (see Figure 2). High-resolution $V V$ intensity images collected by SETHI during the two experiments are shown on Figure 3. In the following, the range axis of images and maps has been dilated compared to the azimuth axis for clarity. Figure 3 (a) depicts the image acquired during the POLLUPROOF'2015 experiment in which the two successive spills are marked off by a yellow ellipse for the rapeseed oil and a red ellipse for the FAME. In between, a mixture of both products is formed and delineated by a blue ellipse [30]. Figure 3 (b) depicts the image acquired during the NOFO'2015 exercise where a feathered structure along the front of the slick and a smooth edge on its back side is observed, which is consistent with the wind direction (green arrow). The crossing of the MOS Sweeper mechanical recovery boom through the slick appears to leave behind a relatively clean sea surface (magenta arrow) and a wake is visible behind the crossing of a ship through the slick (cyan arrow).

\section{B. Results of the quantification method}

The VFE algorithm was applied on both datasets presented in the previous section. Following the steps described in section III, the seawater complex permittivity was estimated from the environmental conditions in Table III and from the central frequency of the SETHI system $\left(f_{E M}=1.325 \mathrm{GHz}\right)$ for both experiments:

$$
\begin{aligned}
\text { POLLUPROOF'2015: } & \varepsilon_{s w} \simeq 72.26+68.71 \mathrm{i} \\
\text { NOFO'2015: } & \varepsilon_{s w} \simeq 74.59+58.26 \mathrm{i}
\end{aligned}
$$

The reference $P R$ of seawater $\left(P R_{S w}\right)$ was estimated by means of a slick-free detection mask and averaged over the maximum of available azimuth pixels. The slick detection mask was estimated by thresholding the Normalized Polarization Difference (NPD) (see [30] for a more detailed description) allowing to separate slick-free and slick-impacted areas. A morphological image processing method (binary opening: binary erosion followed by binary dilation) was applied on the mask in order to remove the last isolated points. The resulting $P R$ s are shown on Figure 4 (a) as a function of the radar range. The corresponding relative roughness parameters on seawater $\left(\Gamma_{s w}\right)$ have been estimated through equation (18) and are depicted on Figure 4 (b).

The different shapes of $\Gamma_{s w}$ parameters (Figure 4 (b)) depend directly on the different shapes of the corresponding $P R \mathrm{~s}$ (Figure 4 (a)), which themselves depend on the acquisition geometry and sea state. The seawater $P R$ s are found of the same order of magnitude as the predicted one on Figure 1 (a), ranging from about 0.35 to 0.4 in the near range $\left(\theta=34^{\circ}\right)$ to about 0.12 in the far range $\left(\theta=52^{\circ}\right)$. A small inflection of the $P R$ is observed at about $3.7 \mathrm{~km}$ and $3.9 \mathrm{~km}$ in range for the POLLUPROOF' 2015 and NOFO'2015 experiments, correspondingly. This effect on the $P R$ is assumed to come from the asymmetrical nature of the observed sea surface, as could be modeled qualitatively by adding a small percentage of asymmetrical wedges to the sea surface slopes distribution [64]. The different locations of the inflection originate from the slightly different azimuth look directions and the different sea states between the two experiments. This inflection is reproduced on the relative roughness parameter $\Gamma_{s w}$ (Figure 4 (b)) as a dip around the same range values. Nevertheless, the high value of the relative roughness parameter $\left(\Gamma_{s w}>0.8\right)$, which is a measure of the deviation from the first order Bragg scattering mechanisms, ensures that Bragg scattering is the dominant mechanism and validates the assumption made in eq. (16) and (17), thus ensuring the enforceability of the VFE algorithm.

The numerical inversion of both the effective complex permittivity $\left(\varepsilon_{e f f}\right)$ and the W/O volume fraction $\left(f_{v}\right)$ has been performed by solving system (19) pixel by pixel on the entire image (avoiding pure seawater areas through the detection mask). The numerical inversion algorithm has been written in Python 3 and executed on the ONERA computational server THOR equipped with 4 x Intel Xeon E7-8867 V3 @ $2.50 \mathrm{GHz}$ and 16 cores, for a total of 64 available computational cores. Parallelization of the code has improved the computation time from about 30 hours to about 25 minutes on this server, in the NOFO'2015 data case. The resulting W/O volume fraction maps and real part of the effective complex permittivity of the two experiments are pictured on Figure 5. Dark gray area represents the slick detection mask and light gray area the ships and SAR processing artifacts removal by means of the co-polarized coherency. 


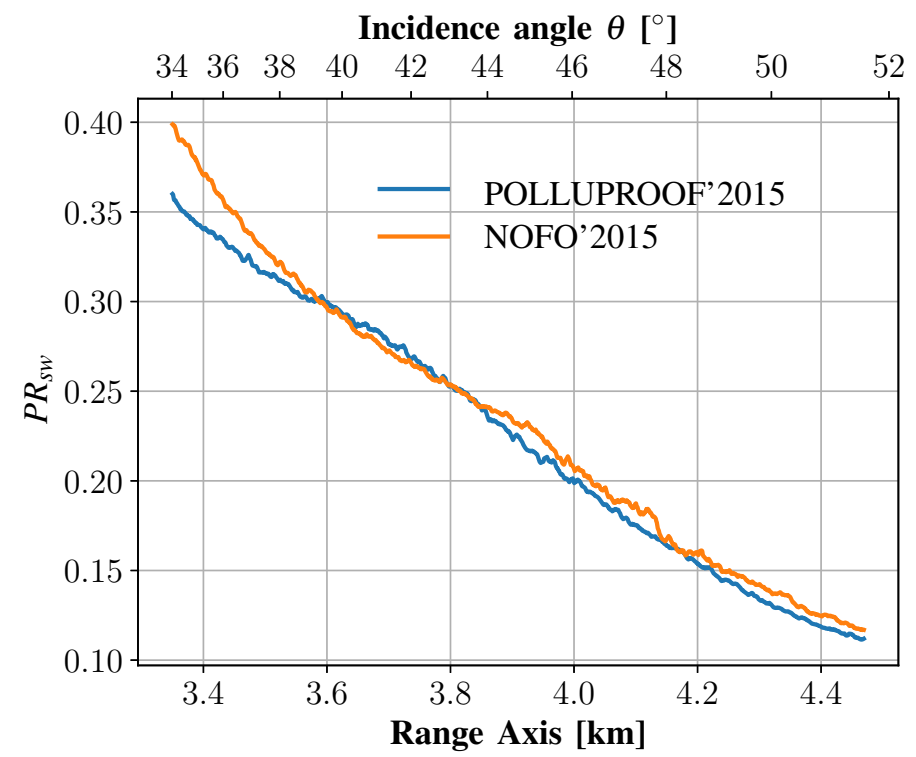

(a) Seawater polarization ratio $P R_{s w}$

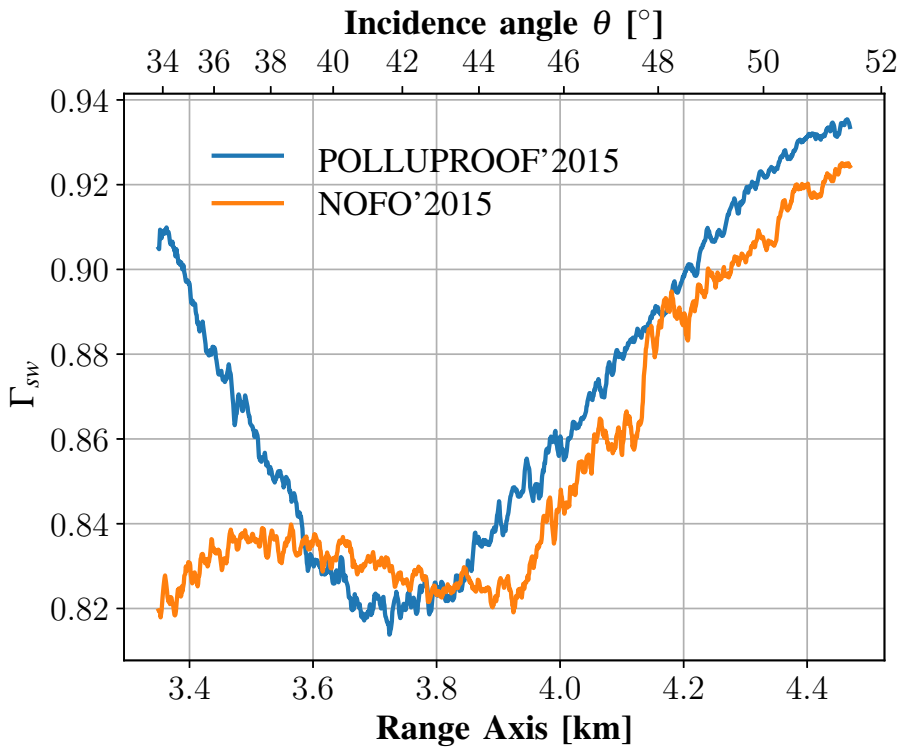

(b) Seawater relative roughness $\Gamma_{s w}$

Figure 4: (a) Estimated mean polarization ratio on seawater from detection mask and (b) the corresponding relative roughness parameter on seawater from POLLUPROOF'2015 (blue curves) and NOFO'2015 (orange curves) L-band SETHI data (see Figure 3).

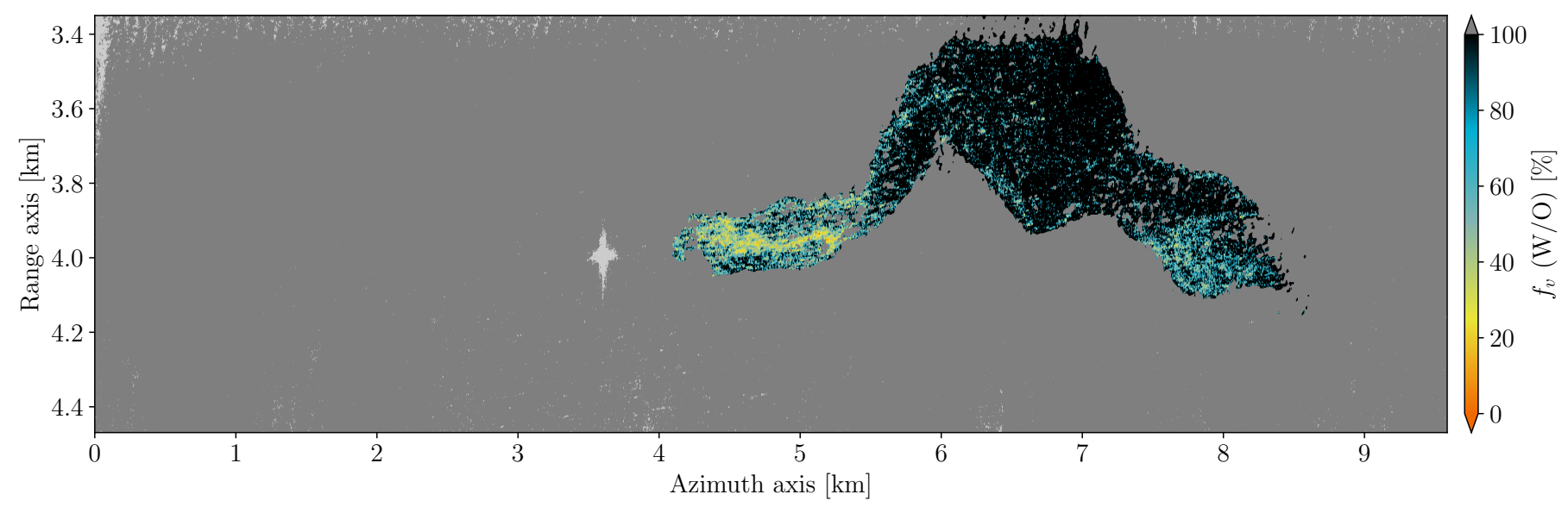

(a) POLLUPROOF'2015

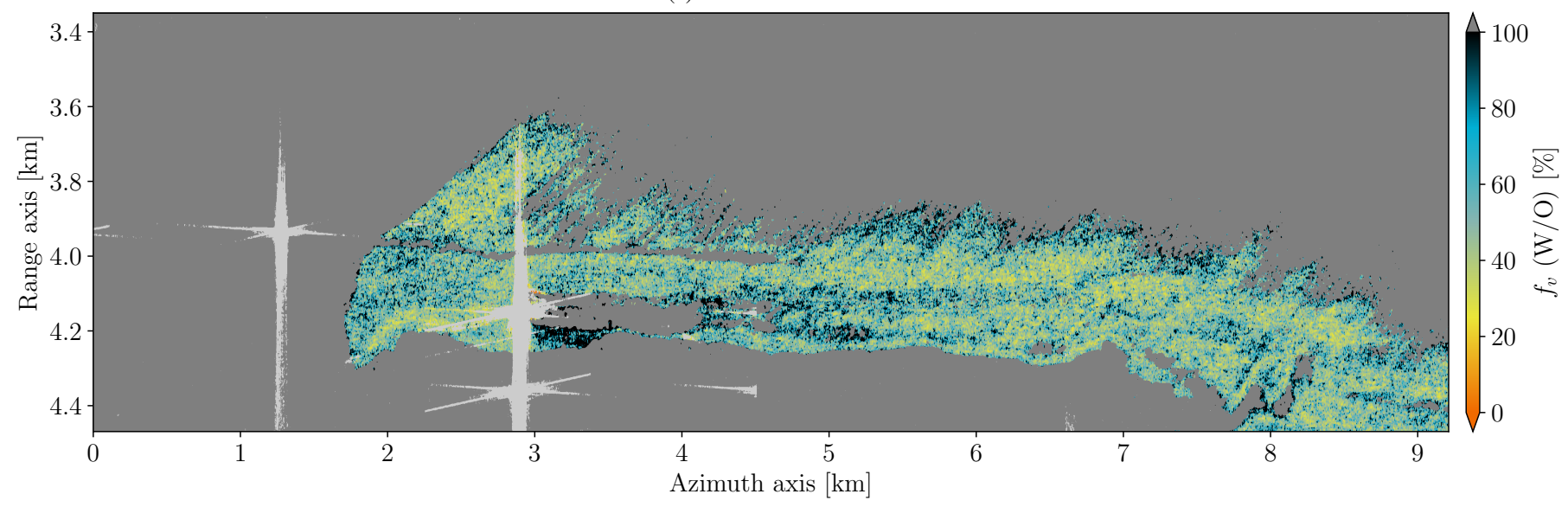

(b) NOFO'2015

Figure 5: Resulting W/O volume fraction maps from VFE algorithm applied to (a) POLLUPROOF'2015 and (b) NOFO'2015 L-band SETHI data (see Figure 3). Dark gray area represents the slick detection mask and light gray area the removal of ships and SAR processing artifacts. 
Figure 5 (a) depicts the resulting W/O volume fraction map estimated from the POLLUPROOF'2015 data. The left part of the slick (FAME release) shows a relatively low W/O volume fraction (yellow color, elevated oil concentration) while the rest of the slick (rapeseed oil release) exhibits a W/O volume fraction close to 1 (black color, very low oil concentration), indicating quasi no mixing of rapeseed oil with seawater, which is consistent with the biogenic behavior of the rapeseed oil. Nevertheless, the very right part of the slick (the beginning of the rapeseed oil release) shows shaded values of $\mathrm{W} / \mathrm{O}$ volume fraction (blue color), indicating the onset of mixing of the rapeseed oil with seawater, likely due to weathering processes induced by wind. Indeed, during the POLLUPROOF' 2015 experiment, a $8.2 \mathrm{~m} . \mathrm{s}^{-1}$ wind speed was recorded (see Table III) which is greater than $5 \mathrm{~m} . \mathrm{s}^{-1}$, the observed upper limit of existence of biogenic slick related to wind speed [9]. Thus indicating that the beginning of the rapeseed oil release is probably being mixed and dissolved in the bulk water. This W/O volume fraction map can be compared to the $M_{\alpha}$ map pictured on [Figure 10 (b), [30]] calculated on the same dataset. Indeed, the estimation of the mixing index $M$ introduced in [28], characterizing the origin of the attenuation of the backscattered signal by the presence of a slick, depends on the difference between two parameters:

$$
M=M_{W}-M_{\alpha},
$$

where the normalized damping factor $M_{W}\left(0 \leq M_{W} \leq 1\right)$ is a measure of the surface roughness damping by the slick $(0$ indicates no damping, 1 a total damping) and the normalized power attenuation factor $M_{\alpha}\left(0 \leq M_{\alpha} \leq 1\right)$ is a measure of the attenuation of the backscattered signal due to a modification of the effective complex permittivity from a mixture ( 0 indicates no attenuation, 1 a total attenuation) [28]. The areas of the impacted backscattered signal from a W/O mixture calculated with the $M_{\alpha}$ parameter depicted on [Figure 10 (b), [30]] are very consistent with the values of the estimated $\mathrm{W} / \mathrm{O}$ volume fraction shown on Figure 5 (a), with the difference that here, a quantitative proportion of $\mathrm{W} / \mathrm{O}$ is provided.

Figure 5 (b) depicts the resulting W/O volume fraction map estimated from the NOFO'2015 data. This map shows an important variation of $\mathrm{W} / \mathrm{O}$ volume fraction within the entire slick. A W/O volume fraction close to 1 (null oil concentration) is seen right behind the MOS Sweeper mechanical recovery boom (around range $4.2 \mathrm{~km}$ and between 3 to $4.5 \mathrm{~km}$ in azimuth) whereas a more graduated variation is observed far behind it, showing the re-forming of the slick due to weathering processes. An increasing value of $\mathrm{W} / \mathrm{O}$ volume fraction is seen in the drag of the slick (top of the image, from yellow to black color) showing a decrease of oil concentration originating from weathering processes.

The resulting W/O volume fraction density functions, calculated from Figure 5 and plotted on Figure 6 show a nonGaussian distribution of W/O volume fractions within both slicks. The density function calculated with the NOFO'2015 data (orange curve) has a narrow peak around about $43 \%$ whereas the density function calculated with the POLLUPROOF'2015 data (blue curve) is much wider. In both cases, the distributions decrease for low value and vanish

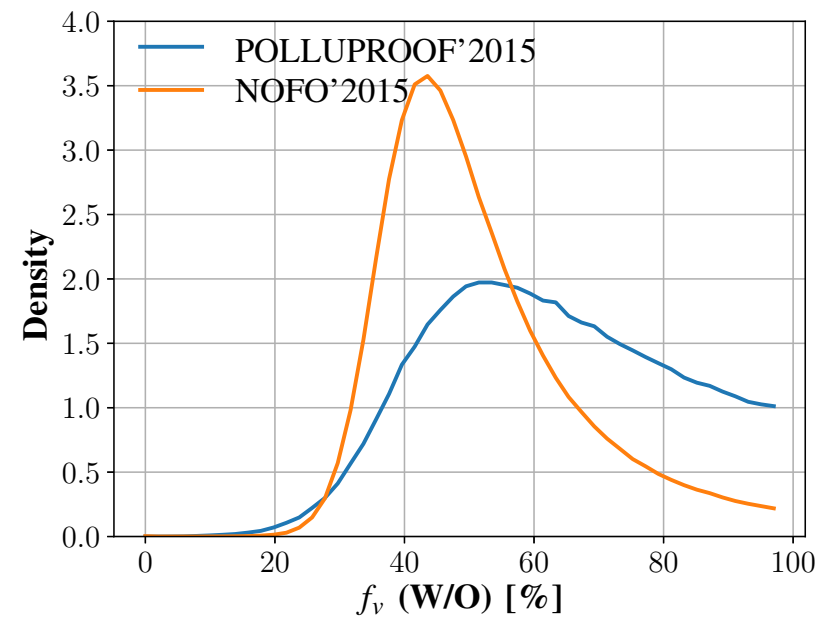

Figure 6: Density functions of estimated W/O volume fractions depicted on Figure 5.

for W/O volume fractions lower than about $20 \%$. At large values, the NOFO'2015 distribution slightly decreases to the pure seawater case $\left(f_{v}=100 \%\right)$, while on the contrary, the POLLUPROOF' 2015 distribution remains elevated in the pure seawater case, which is consistent with the presence of the rapeseed oil film in the slick.

\section{CONClusion}

We presented a marine oil slicks quantification method to estimate the volume fraction, together with the complex effective permittivity, of a water-in-oil mixture from L-band dual-polarization SAR imagery. The quantification method is based on the physical modeling of both oil/seawater and electromagnetic waves/sea surface interactions. The Universal Weighted Curvature Approximation scattering model was chosen as a good trade-off between a relevant description of the polarimetric parameters and simple and versatile formulation well adapted to the inversion of oceanic features. An inversion algorithm has been derived, namely, the Volume Fraction Estimation (VFE) algorithm, for the study of volume scattering effects. It uses the L-band Polarization Ratio properties and allows one to estimate the effective complex permittivity of the observed surface in addition to the W/O volume fraction. The enforceability of the algorithm is ensured through the high value of the relative roughness parameter $(\Gamma>0.8)$, showing that Bragg scattering is the dominant mechanism. Therefore, this algorithm can solely be applied to L-band SAR data which also have very low noise level. These requirements are reached by actual airborne sensors such as the American UAVSAR or the French SETHI systems but the use with satellite data, such as the Japanese L-band ALOS system, would be limited by the instrument noise level. A first application of the VFE algorithm has been made to L-band dual-polarized experimental data recorded during the POLLUPROOF'2015 and NOFO'2015 experiments with the ONERA airborne SETHI system, leading to a very consistent map of the W/O volume fraction. This kind of oil concentration map could be used in different manners and for different field of applications. For instance, 
in environmental monitoring, it could be used to concentrate cleaning efforts to the most oil-concentrated area in the case of accidental oil spill. Oil concentration map could also be used for temporal monitoring of oil concentration in the survey of natural oil seeps or other marine oil slicks.

\section{ACKNOWLEDGMENT}

Research presented in this paper is part of the NAOMI (New Advanced Observation Method Integration) Project funded by Total (the French petroleum company) and ONERA (the French Aerospace Lab). The authors would like to thank all people involved in this project, and especially PierreYves Foucher, Véronique Miegebielle and Dominique Dubucq for supporting this work. A special thank goes to ONERA's engineer Joseph Martinot-Lagarde for his very useful help in Python code parallelization. The authors are very grateful to the NOFO (Norwegian Clean Seas Association for Operating Companies) for allowing us to participate in the Oil-on-Water exercise, which was carried out during the period 8-14 June, 2015.

\section{REFERENCES}

[1] I. Leifer, W. J. Lehr, D. Simecek-Beatty, E. Bradley, R. Clark, P. Dennison, Y. Hu, S. Matheson, C. E. Jones, B. Holt, M. Reif, D. A. Roberts, J. Svejkovsky, G. Swayze, and J. Wozencraft, "State of the art satellite and airborne marine oil spill remote sensing: Application to the bp deepwater horizon oil spill," Remote Sensing of Environment, vol. 124, no. Supplement C, pp. $185-209,2012$.

[2] C. Brekke and A. Solberg, "Oil spill detection by satellite remote sensing," Remote Sensing of Environment, vol. 95, no. 1, pp. 1 - 13, 2005.

[3] M. Fingas and C. Brown, "Review of oil spill remote sensing," Marine Pollution Bulletin, vol. 83, no. 1, pp. 9 - 23, 2014.

[4] R. Jatiault, D. Dhont, L. Loncke, and D. Dubucq, "Monitoring of natural oil seepage in the lower congo basin using sar observations," Remote Sensing of Environment, vol. 191, no. Supplement C, pp. 258 - 272, 2017.

[5] M. Gade and W. Alpers, "Using ers-2 sar images for routine observation of marine pollution in european coastal waters," Science of The Total Environment, vol. 237-238, no. Supplement C, pp. 441 - 448, 1999.

[6] F. Girard-Ardhuin, G. Mercier, F. Collard, and R. Garello, "Operational oil-slick characterization by sar imagery and synergistic data," IEEE Journal of Oceanic Engineering, vol. 30, pp. 487-495, July 2005.

[7] O. Garcia-Pineda, B. Zimmer, M. Howard, W. Pichel, X. Li, and I. MacDonald, "Using sar images to delineate ocean oil slicks with a texture-classifying neural network algorithm (tcnna)," Canadian Journal of Remote Sensing, vol. 35, no. 5, pp. 411-421, 2009.

[8] M. Gade, H. Hühnerfuss, and G. Korenowski, Marine Surface Films: Chemical Characteristics, Influence on Air-Sea Interactions and Remote Sensing. Springer, Berlin, Heidelberg, 2006.

[9] J.-C. Romano, "Sea-surface slick occurrence in the open sea (mediterranean, red sea, indian ocean) in relation to wind speed," Deep Sea Research Part I: Oceanographic Research Papers, vol. 43, no. 4, pp. 411 - 423, 1996.

[10] S. Mudge, M. Salgado, and J. East, "Preliminary investigations into sunflower oil contamination following the wreck of the m.v. kimya," Marine Pollution Bulletin, vol. 26, no. 1, pp. 40 - 44, 1993.

[11] S. M. Mudge, "Deleterious effects from accidental spillages of vegetable oils," Spill Science \& Technology Bulletin, vol. 2, no. 2, pp. 187 - 191, 1995.

[12] P. Rajinder, "Effect of droplet size on the rheology of emulsions," $A I C h E$ Journal, vol. 42, no. 11, pp. 3181-3190, 1996.

[13] M. Fingas and B. Fieldhouse, "Studies on water-in-oil products from crude oils and petroleum products," Marine Pollution Bulletin, vol. 64, no. 2, pp. $272-283,2012$.

[14] V. G. Levich, Physicochemical hydrodynamics. Englewood Cliffs, N.J: Prentice-Hall, 1962.
[15] R. Cini and P. P. Lombardini, "Damping effect of monolayers on surface wave motion in a liquid," Journal of Colloid and Interface Science, vol. 65 , no. 2 , pp. $387-389,1978$.

[16] J. Lucassen, "Effect of surface-active material on the damping of gravity waves: A reappraisal," Journal of Colloid and Interface Science, vol. 85, no. 1 , pp. $52-58,1982$.

[17] S. Ermakov, A. Zujkova, A. Panchenko, S. Salashin, T. Talipova, and V. Titov, "Surface film effect on short wind waves," Dynamics of Atmospheres and Oceans, vol. 10, no. 1, pp. $31-50,1986$.

[18] H. Hühnerfuss, W. Alpers, H. Dannhauer, M. Gade, P. A. Lange, V. Neumann, and V. Wismann, "Natural and man-made sea slicks in the north sea investigated by a helicopter-borne 5-frequency radar scatterometer," International Journal of Remote Sensing, vol. 17, no. 8, pp. $1567-1582,1996$.

[19] M. Gade, W. Alpers, H. Hühnerfuss, and P. A. Lange, "Wind wave tank measurements of wave damping and radar cross sections in the presence of monomolecular surface films," Journal of Geophysical Research: Oceans, vol. 103, no. C2, pp. 3167-3178, 1998.

[20] W. Alpers and H. Hühnerfuss, "Radar signatures of oil films floating on the sea surface and the marangoni effect," Journal of Geophysical Research: Oceans, vol. 93, no. C4, pp. 3642-3648, 1988.

[21] V. Wismann, M. Gade, W. Alpers, and H. Hühnerfuss, "Radar signatures of marine mineral oil spills measured by an airborne multi-frequency radar," International Journal of Remote Sensing, vol. 19, no. 18, pp. 3607-3623, 1998 .

[22] M. Gade, W. Alpers, H. Hühnerfuss, V. R. Wismann, and P. A. Lange, "On the reduction of the radar backscatter by oceanic surface films: Scatterometer measurements and their theoretical interpretation," Remote Sensing of Environment, vol. 66, no. 1, pp. 52 - 70, 1998.

[23] A. H. S. Solberg, "Remote sensing of ocean oil-spill pollution," Proceedings of the IEEE, vol. 100, pp. 2931-2945, Oct 2012.

[24] M. Migliaccio, F. Nunziata, and A. Buono, "Sar polarimetry for sea oil slick observation," International Journal of Remote Sensing, vol. 36, no. 12 , pp. 3243-3273, 2015.

[25] S. Angelliaume, P. Dubois-Fernandez, C. E. Jones, B. Holt, B. Minchew, E. Amri, and V. Miegebielle, "Sar imagery for detecting sea surface slicks: Performance assessment of polarization-dependent parameters," IEEE Transactions on Geoscience and Remote Sensing, in press, 2018.

[26] S. Skrunes, C. Brekke, and T. Eltoft, "Characterization of marine surface slicks by radarsat-2 multipolarization features," IEEE Transactions on Geoscience and Remote Sensing, vol. 52, pp. 5302-5319, Sept 2014.

[27] S. Skrunes, C. Brekke, T. Eltoft, and V. Kudryavtsev, "Comparing nearcoincident c- and $\mathrm{x}$-band sar acquisitions of marine oil spills," IEEE Transactions on Geoscience and Remote Sensing, vol. 53, pp. 19581975, April 2015.

[28] B. Minchew, "Determining the mixing of oil and sea water using polarimetric synthetic aperture radar," Geophysical Research Letters, vol. 39 , no. 16, pp. n/a-n/a, 2012. L16607.

[29] B. Minchew, C. E. Jones, and B. Holt, "Polarimetric analysis of backscatter from the deepwater horizon oil spill using L-band synthetic aperture radar," IEEE Transactions on Geoscience and Remote Sensing, vol. 50, pp. 3812-3830, Oct 2012.

[30] S. Angelliaume, B. Minchew, S. Chataing, P. Martineau, and V. Miegebielle, "Multifrequency radar imagery and characterization of hazardous and noxious substances at sea," IEEE Transactions on Geoscience and Remote Sensing, vol. 55, pp. 3051-3066, May 2017.

[31] T. Elfouhaily, B. Chapron, K. Katsaros, and D. Vandemark, "A unified directional spectrum for long and short wind-driven waves," Journal of Geophysical Research, vol. 102, no. C7, pp. 15781-15796, 1997.

[32] T. Meissner and F. J. Wentz, "The complex dielectric constant of pure and sea water from microwave satellite observations," IEEE Transactions on Geoscience and Remote Sensing, vol. 42, pp. 1836-1849, Sept 2004.

[33] N. Aske, R. Orr, and J. Sjöblom, "Dilatational elasticity moduli of water-crude oil interfaces using the oscillating pendant drop," Journal of Dispersion Science and Technology, vol. 23, no. 6, pp. 809-825, 2002.

[34] M. H. Sharqawy, J. H. Lienhard V, and S. M. Zubair, "Thermophysical properties of seawater: A review of existing correlations and data," Desalination and Water Treatment, vol. 16, pp. 354-380, April 2010.

[35] C. Marangoni, "Sul principio della viscosità superficiale dei liquidi stabilito dal sig. J. Plateau," Nuovo Cimento, vol. Series 2, Vol. 5/6, p. 239, 1872.

[36] W. Alpers and H. Hühnerfuss, "The damping of ocean waves by surface films: A new look at an old problem," Journal of Geophysical Research: Oceans, vol. 94, no. C5, pp. 6251-6265, 1989.

[37] A. D. Jenkins and S. J. Jacobs, "Wave damping by a thin layer of viscous fluid," Physics of Fluids, vol. 9, no. 5, pp. 1256-1264, 1997. 
[38] P. P. Lombardini, B. Fiscella, P. Trivero, C. Cappa, and W. D. Garrett, "Modulation of the spectra of short gravity waves by sea surface films: Slick detection and characterization with a microwave probe," Journal of Atmospheric and Oceanic Technology, vol. 6, no. 6, pp. 882-890, 1989.

[39] K. Folgerø, "Bilinear calibration of coaxial transmission/reflection cells for permittivity measurement of low-loss liquids," Measurement Science and Technology, vol. 7, no. 9, pp. 1260-1269, 1996.

[40] T. Friisø, Y. Schildberg, O. Rambeau, T. Tjomsland, H. Førdedal, an J. Sjøblom, "Complex permittivity of crude oils and solutions of heavy crude oil fractions," Journal of Dispersion Science and Technology, vol. 19, no. 1, pp. 93-126, 1998.

[41] A. Sihvola, Electromagnetic Mixing Formulas and Applications. Electromagnetic Waves, Institution of Engineering and Technology, 1999.

[42] C.-A. Guérin and A. Sentenac, "Separation of surface and volume effects in scattering from heterogeneous rough surfaces: derivation of a splitting rule," Journal of the Optical Society of America A, vol. 24, pp. 385-390, Feb 2007.

[43] T. Elfouhaily and C.-A. Guérin, "A critical survey of approximate scattering wave theories from random rough surfaces," Waves in Random Media, vol. 14, no. 4, pp. R1-R40, 2004.

[44] S. O. Rice, "Reflection of electromagnetic waves from slightly rough surfaces.," Communications on Pure and Applied Mathematics, vol. 4 no. 2-3, pp. 351-378, 1951

[45] G. R. Valenzuela, "Theories for the interaction of electromagnetic an oceanic waves - a review," Boundary-Layer Meteorology, vol. 13, no. 1, pp. 61-85, 1978.

[46] P. Beckmann and A. Spizzichino, The scattering of electromagnetic waves from rough surfaces. Artech House Radar Library, 1963.

[47] A. G. Voronovich, "Small-slope approximation in wave scattering from rough surfaces.," Journal of Experimental and Theoretical Physics, vol. 62 , no. 1 , pp. $65-70,1985$

[48] A. G. Voronovich, "Small-slope approximation for electromagnetic wave scattering at a rough interface of two dielectric half-spaces.," Waves in Random and Complex Media, vol. 4, no. 3, pp. 337-367, 1994.

[49] A. G. Voronovich, Wave scattering from rough surfaces. Springer Berlin Heidelberg, 1994.

[50] J. Wright, "A new model for sea clutter," IEEE Transactions on Antennas and Propagation, vol. 16, pp. 217-223, March 1968.

[51] G. Soriano and C.-A. Guérin, "A cutoff invariant two-scale model in electromagnetic scattering from sea surfaces," IEEE Geoscience and Remote Sensing Letters, vol. 5, pp. 199-203, April 2008

[52] B. Chapron, V. Kerbaol, D. Vandemark, and T. Elfouhaily, "Importance of peakedness in sea surface slope measurements and applications," Journal of Geophysical Research: Oceans, vol. 105, no. C7, pp. 1719517202,2000

[53] T. Elfouhaily, S. Guignard, R. Awadallah, and D. R. Thompson, "Local and non-local curvature approximation: a new asymptotic theory for wave scattering," Waves in Random Media, vol. 13, no. 4, pp. 321-337, 2003.

[54] C.-A. Guérin, G. Soriano, and T. Elfouhaily, "Weighted curvature approximation: numerical tests for $2 \mathrm{~d}$ dielectric surfaces," Waves in Random Media, vol. 14, no. 3, pp. 349-363, 2004

[55] A. A. Mouche, B. Chapron, and N. Reul, "A simplified asymptotic theory for ocean surface electromagnetic wave scattering," Waves in Random and Complex Media, vol. 17, no. 3, pp. 321-341, 2007.

[56] A. A. Mouche, B. Chapron, N. Reul, D. Hauser, and Y. Quilfen, "Importance of the sea surface curvature to interpret the normalized radar cross section," Journal of Geophysical Research: Oceans, vol. 112, no. C10, pp. n/a-n/a, 2007. C10002.

[57] C.-A. Guerin, G. Soriano, and B. Chapron, "The weighted curvature approximation in scattering from sea surfaces," Waves in Random and Complex Media, vol. 20, no. 3, pp. 364-384, 2010.

[58] G. Caulliez and C.-A. Guérin, "Higher-order statistical analysis of short wind wave fields," Journal of Geophysical Research: Oceans, vol. 117 no. C6, pp. n/a-n/a, 2012. C06002.

[59] S. Angelliaume, X. Ceamanos, F. Viallefont-Robinet, R. Baqué, P. Déliot, and V. Miegebielle, "Hyperspectral and radar airborne imagery over controlled release of oil at sea," Sensors, vol. 17, no. 8, 1772, 2017.

[60] M. Gade, W. Alpers, H. Hühnerfuss, H. Masuko, and T. Kobayashi, "Imaging of biogenic and anthropogenic ocean surface films by the multifrequency/multipolarization sir-c/x-sar," Journal of Geophysical Research: Oceans, vol. 103, no. C9, pp. 18851-18866, 1998.

[61] W. Alpers, B. Holt, and K. Zeng, "Oil spill detection by imaging radars: Challenges and pitfalls," Remote Sensing of Environment, vol. 201, pp. $133-147,2017$
[62] "MOS Sweeper - Egersund Group." http://www.egersundgroup.no/ oilspill/mos-sweeper-. Accessed: 2017-10-23.

[63] I. Hajnsek, E. Pottier, and S. R. Cloude, "Inversion of surface parameters from polarimetric sar," IEEE Transactions on Geoscience and Remote Sensing, vol. 41, pp. 727-744, April 2003.

[64] Z. Guerraou, Rétrodiffusion micro-onde par la surface océanique en incidence élevée : approche conjointe théorique et expérimentale. $\mathrm{PhD}$ thesis, Université de Toulon, France, September 2017. In French.

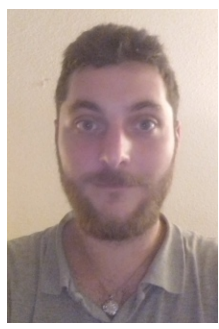

Olivier Boisot received the M.S. degree in physics and engineering sciences in 2012 and the $\mathrm{Ph} . \mathrm{D}$. degree in physics in 2015 , specialized in ocean remote sensing, from the University of Toulon, La Garde, France. He worked as a Postdoctoral Fellow within the Electromagnetism and Radar Department of the Office National d'Études et de Recherches Aérospatiales (ONERA), Salon-de-Provence, France, on the marine oil slicks quantification from Synthetic Aperture Radar (SAR) measurements in microwave bands.

He is currently working within the ONERA on SAR interferometry capabilities over the ocean surface.

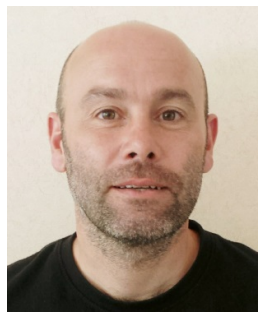

Sébastion Angelliaume received the Engineering degree from the École Nationale Supérieure d'Ingénieur de Constructions Aéronautiques, Toulouse, France, and the M.S. degree from the École Nationale Supérieure d'Électrotechnique, d'Électronique, d'Informatique, d'Hydraulique et des Télécommunications, Toulouse, both in 2003. Since 2006, he has been with the Electromagnetism and Radar Department at the Office National d'Études et de Recherches Aérospatiales (ONERA - The French Aerospace Lab), Salon de Provence, France. He has been involved in the ONERA synthetic aperture radar airborne platform RAMSES and SETHI, developing science applications. Since 2010, his research at ONERA focuses mainly on remote sensing over the ocean surface.

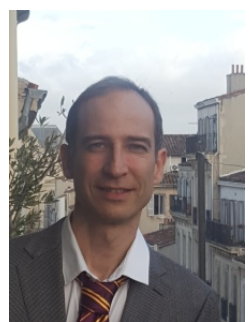

Charles-Antoine Guérin received the B. Eng. degree from the École Nationale Supérieure de l'Aéronautique et de l'Espace, Toulouse, France, in 1994 and the Ph.D. degree in Theoretical Physics from the University of Aix-Marseille, France, in 1998

He is now professor and researcher at the University of Toulon, France, with the Mediterranean Institute of Oceanography. He is specialized in ocean remote sensing. 